\title{
Perspective
}

\section{Peptide nucleic acid antisense oligomers open an avenue for developing novel antibacterial molecules}

\author{
Anubrata Ghosal \\ Department of Biology, Massachusetts Institute of Technology, Cambridge, MA, United States
}

Key words: PNA; antibacterial; antisense; Pseudomonas

J Infect Dev Ctries 2017; 11(2):212-214. doi:10.3855/jidc.9159

(Received 14 July 2016 - Accepted 12 September 2016)

Copyright (C) 2017 Ghosal This is an open-access article distributed under the Creative Commons Attribution License, which permits unrestricted use, distribution, and reproduction in any medium, provided the original work is properly cited.

Bacterial drug resistance is a growing concern around the globe. The indiscriminate use of antibiotics in many parts of the world for decades has helped bacteria to acquire resistance against commonly used antibiotics. This situation is worsened further due to transfer of resistance gene(s) within bacteria, promoting the rise of multidrug resistant bacterial strains.

Multidrug resistance in Pseudomonas aeruginosa is also a growing concern. This bacterium is considered as an opportunistic human pathogen and known to infect patients with suppressed immunity. Pseudomonas can grow in a wide variety of hosts, ranging from plants to animals $[1,2]$. It is also found in wetlands, soil, and marine environments, and its ability to acquire resistance against antibiotics and disinfectants makes $P$. aeruginosa different from other bacteria [3]. Several hundreds of people die each year due to Pseudomonas infection. In most cases, this nosocomial pathogen is responsible for the death of cystic fibrosis patients.

$P$. aeruginosa has a comparatively large genome of 6.3 million bases that encodes 5570 proteins [3]. Large genetic diversity enhances $P$. aeruginosa ability to gain resistance against antibiotics quickly and even during the treatment. Several $P$. aeruginosa clinical isolates are resistant to different classes of antibiotics, for example, $\quad \beta$-lactams, fluoroquinolones, and aminoglycosides [4,5]. Resistance to $\beta$-lactam and aminoglycoside classes of antibiotics is due to the import of genetic material, whereas resistance to fluoroquinolones is linked to mutations [4]. $P$. aeruginosa acquires resistance against antibiotics either by modification of the target or overexpression of the efflux pump or alteration of the membrane permeability $[6,7]$.
Multidrug resistant bacterial infections pose a significant challenge in the treatment of patients. Currently, we are left with few treatment options. The reduced arsenal of antibiotics is pushing us to a postantibiotic era. The long discovery time and a huge development-associated cost limit the discovery of new antibacterial compounds. The majority of effective antibiotics are derivatives of earlier discovered antibiotics. We are in need of new classes of antibiotics with novel mechanism of action.

About one and a half decade ago, oligomers of Peptide Nucleic Acid (PNA) were first introduced as antibacterial agents, and studies showed that these antibacterial PNA molecules are capable of inhibiting the growth of outer-membrane defective E. coli strain $[8,9]$. Years later, a separate study exhibited that PNA oligomer in conjugation with KFFKFFKFFK peptide is also able to inhibit the growth of wild type $E$. coli strain [10]. Since then, numerous studies have reported the antibacterial activity of peptide-PNA conjugates in different bacterial strains, Campylobacter jejuni, Staphylococcus aureus, Mycobacterium smegmatis, Brucella suis, Pseudomonas aeruginosa, Klebsiella pneumonia, E. coli, Shigella flexneri, and Streptococcus pyogenes [11-20].

PNA is a synthetic molecule that mimics the structure of DNA. Synthesis of PNA oligomers follows a simple chemical approach, which has been well described in the literature [21]. Inside the cell, these antibacterial PNA oligomers function as antisense molecules and initiate inhibition of translation either by blocking the movement of ribosomes along the mRNA or disrupting the assembly of ribosome around the translation start site. The interaction between the PNA 
oligomer and mRNA is reversible and governed by hydrogen bonds. Designing PNA oligomers is relatively straightforward, but it requires a systematic search to identify the optimal target region on the mRNA in order to achieve maximum inhibition of gene expression. Generally, the area around the translation start site -20 nucleotides up- and downstream - is optimal for designing antibacterial PNA molecules [15]. However, factors such as RNA secondary structure and association of RNA-binding proteins with RNA, could make the designing of antibacterial PNA oligomers challenging. Regions other than the one around the translation start site could also be important for antibacterial PNA design. In the study by Ghosal et al. [15], antibacterial molecules were designed through steps that involve identification of target, scanning of regions on mRNA, characterization of PNA oligomers length, and, lastly, validation of carrier peptides. These steps described in detail in the literature [10].

Four antibacterial peptide-PNA conjugates were discovered and developed in the study by Ghosal et al. [15]. These molecules efficiently inhibit $P$. aeruginosa growth at low micromolar concentrations and are effective against three strains of $P$. aeruginosa, namely, PA01 (a commonly used laboratory stain), PA14 (a highly virulent strain) and LESB58 (a clinical isolate from a cystic fibrosis patient). Two of these four molecules, target the mRNA of acpP gene. The target region of anti-acpP PNA is conserved in a range of species of Pseudomonas, highlighting the potential antibacterial application of anti-acpP conjugates in other Pseudomonas species.

PNA is a synthetic molecule and not found in any living systems, while number of commonly used antibiotics are natural in origin. Therefore, it is likely that in the course of evolution, bacteria may not have a chance to encounter PNA molecules, so one could assume that gaining resistance against PNA molecules will be difficult for bacteria.

Furthermore, the selective killing of pathogenic bacteria is a big challenge. The majority of antibiotics negatively affects the host-associated beneficial bacterial community. Designing of bacterial-specific antibiotics is significantly challenging while it is relatively simpler using the PNA-based approach. The delivery of antibacterial PNA molecules can be achieved by designing of bacterial-specific vehicles. Several peptides, such as $(\mathrm{KFF})_{3} \mathrm{~K}$, (R-Ahx-R)4-Ahxßala, (R-Ahx)6-ßala, YARVRRRGPRGYARVRRRGPRRC, and RFFRFFRFFRXB, are known for delivering antisense PNA or Morpholino oligomers in bacteria
$[10,15,22,23]$. In addition to their structural and/or sequence dissimilarity, these peptides may also differ in their uptake in bacteria.

Certain modifications can also alter the entry of peptide-PNA conjugates in bacteria. For instance, the addition of a cleavable linker, -F-Gly-eg1- or -FFKeg1-, between peptide and PNA oligomer allows PNA oligomer to separate from the conjugated peptide in the bacterial periplasm [17]. Additionally, a study on $E$. coli highlighted that the $\mathrm{L}$ form of $(\mathrm{KFF})_{3} \mathrm{~K}$ peptide degrades in periplasm while $\mathrm{H}-\mathrm{D}\left((\mathrm{KFF})_{3} \mathrm{~K}\right)$, (R-AhxR)4-Ahx- $\beta$ ala and (R-Ahx)6- $\beta$ ala peptides remain attached to the PNA oligomer and assist delivery of PNA oligomers to the bacterial cytoplasm [17]. Moreover, the uptake of these cell permeable peptides varies among bacteria, for example, $(\mathrm{KFF})_{3} \mathrm{~K}$ peptide transports PNA oligomer efficiently in E. coli but not in $P$. aeruginosa [15].

It is equally important that these delivery peptides remain stable and effective in the mammalian system. It has been shown that PNA or Morpholino antisense oligomers in conjugation with $(\mathrm{KFF})_{3} \mathrm{~K},(\mathrm{RX})_{6} \mathrm{~B}-$, $(\mathrm{RXR})_{4} \mathrm{XB}-$, and (RFR) ${ }_{4} \mathrm{XB}$ peptides are able to eliminate bacterial infection in mice [18,24-26]. Furthermore, it is also possible that the peptides which efficiently transport PNA oligomers in mammalian cells may also be capable of transporting PNA oligomers in bacteria [27, 28].

Additionally, antisense PNA oligomers could also be used for studying gene function in bacteria. Following observations strongly highlight that the development of PNA antibiotics could be an alternative approach to control multidrug resistance bacterial infections.

\section{References}

1. Walker TS, Bais HP, Deziel E, Schweizer HP, Rahme LG, Fall R, Vivanco JM (2004) Pseudomonas aeruginosa-plant root interactions. Pathogenicity, biofilm formation, and root exudation. Society 134: 320-331.

2. Mahajan-Miklos S, Tan MW, Rahme LG, Ausubel FM (1999) Molecular mechanisms of bacterial virulence elucidated using a Pseudomonas aeruginosa-Caenorhabditis elegans pathogenesis model. Cell 96: 47-56.

3. Stover CK, Pham XQ, Erwin AL, Mizoguchi SD, Warrener P, Hickey MJ, Brinkman FS, Hufnagle WO, Kowalik DJ, Lagrou M, Garber RL, Goltry L, Tolentino E, Westbrock-Wadman S, Yuan Y, Brody LL, Coulter SN, Folger KR, Kas A, Larbig K, Lim R, Smith K, Spencer D, Wong GK, Wu Z, Paulsen IT, Reizer J, Saier MH, Hancock RE, Lory S, Olson MV (2000) Complete genome sequence of Pseudomonas aeruginosa PAO1, an opportunistic pathogen. Nature 406: 959-964.

4. Lister PD, Wolter DJ, Hanson ND (2009) Antibacterialresistant Pseudomonas aeruginosa: clinical impact and complex regulation of chromosomally encoded resistance mechanisms. Clin Microbiol Rev 22: 582-610. 
5. Livermore DM (2002) Multiple mechanisms of antimicrobial resistance in Pseudomonas aeruginosa: our worst nightmare? Clin Infect Dis 34: 634-640.

6. Porras-Gómez M, Vega-Baudrit J, Núñez-Corrales S (2012) Overview of multidrug-resistant Pseudomonas aeruginosa and novel therapeutic approaches. J Biomater Nanobiotechnol 03: 519-527..

7. Allen HK, Donato J, Wang HH, Cloud-Hansen KA, Davies J, Handelsman J (2010) Call of the wild: antibiotic resistance genes in natural environments. Nat Rev Microbiol. 8: 251259.

8. Good L, Nielsen PE (1998) Inhibition of translation and bacterial growth by peptide nucleic acid targeted to ribosomal RNA. Proc Natl Acad Sci USA 95: 2073-2076.

9. Good L, Nielsen PE (1998) Antisense inhibition of gene expression in bacteria by PNA targeted to mRNA. Nat Biotechnol 16: 355-358.

10. Good L, Awasthi SK, Dryselius R, Larsson ONP (2001) Bactericidal antisense effects of peptide-PNA conjugates. Nat Biotechnol 19: 360-364.

11. Jeon BZQ (2009) Sensitization of Campylobacter jejuni to fluoroquinolone and macrolide antibiotics by antisense inhibition of the CmeABC multidrug efflux transporter. J Antimicrob Chemother 63: 946-948.

12. Nekhotiaeva N, Awasthi SK, Nielsen PE and GL (2004) Inhibition of Staphylococcus aureus gene expression and growth using antisense peptide nucleic acids. Mol Ther 10: 652-659.

13. Kulyté A, Nekhotiaeva N, Awasthi SKGL (2005) Inhibition of Mycobacterium smegmatis gene expression and growth using antisense peptide nucleic acids. J Mol Microbiol Biotechnol 9: 101-109.

14. Rajasekaran P, Alexander JC, Seleem MN, Jain N, Sriranganathan N, Wattam AR, Setubal JC, Boyle SM (2013) Peptide nucleic acids inhibit growth of Brucella suis in pure culture and in infected murine macrophages. Int $\mathbf{J}$ Antimicrob Agents 41: 358-362.

15. Ghosal A, Nielsen PE (2012) Potent antibacterial antisense peptide-peptide nucleic acid conjugates against Pseudomonas aeruginosa. Nucleic Acid Ther 22: 323-334.

16. Kurupati P, Tan KS, Kumarasinghe GPC (2007) Inhibition of gene expression and growth by antisense peptide nucleic acids in a multiresistantbeta-lactamase-producing Klebsiella pneumoniae strain. Antimicrob Agents Chemother 51: 805811.

17. Ghosal A, Vitali A, Stach JEM, Nielsen PE (2013) Role of SbmA in the uptake of peptide nucleic acid (PNA)-peptide conjugates in E. coli. ACS Chem Biol 8: 360-367.

18. Bai H, You Y, Yan H, Meng J, Xue X, Hou Z, Zhou Y, Ma X, Sang GLX (2012) Antisense inhibition of gene expression and growth in gram-negative bacteria by cell-penetrating peptide conjugates of peptide nucleic acids targeted to $\mathrm{rpoD}$ gene. Biomaterials 33: 659-667.

19. Patenge N, Pappesch R, Krawack F, Walda C, Mraheil MA, Jacob A, Hain T, Kreikemeyer B (2013) Inhibition of growth and gene expression by PNA-peptide conjugates in Streptococcus pyogenes. Mol Ther Nucleic Acids 2: e132.

20. Ghosal A (2013) Novel antibacterial agents (antibiotics) based on RNA interference using Peptide Nucleic Acid (PNA). Doctorate thesis in Health Science. University of Copenhagen. Copenhagen- Hovedstaden. 107p.

21. Braasch DA, Nulf CJ, Corey DR (2002) Synthesis and purification of peptide nucleic acids. Curr Protoc Nucleic Acid Chem. 4: 1-18.

22. Greenberg DE, Marshall-Batty KR, Brinster LR, Zarember KA, Shaw PA, Mellbye BL, Iversen PL, Holland SM, Geller BL (2010) Marshall-antisense phosphorodiamidate morpholino oligomers targeted to an essential gene inhibit Burkholderia cepacia complex. J Infect Dis 201: 1822-1830.

23. Wesolowski D, Tae HS, Gandotra N, Llopis P, Shen NAS (2011) Basic peptidemorpholino oligomer conjugate that is very effective in killing bacteria by gene-specific and nonspecific modes. Proc Natl Acad Sci USA 108: 16582 16587.

24. Tan XX, Actor JKCY (2005) Peptide nucleic acid antisense oligomer as a therapeutic strategy against bacterial infection: proof of principle using mouse intraperitoneal infection. Antimicrob Agents Chemother 49: 3203-3207.

25. Mellbye BL, Puckett SE, Tilley LD, Iversen PL, Geller BL (2009) Variations in amino acid composition of antisense peptide-phosphorodiamidate morpholino oligomer affect potency against Escherichia coli in vitro and in vivo. Antimicrob Agents Chemother 55: 525-530.

26. Mellbye BL, Weller DD, Hassinger JN, Reeves MD, Lovejoy CE, Iversen PL GB (2010) Cationic phosphorodiamidate morpholino oligomers efficiently prevent growth of Escherichia coli in vitro and in vivo. J Antimicrob Chemother 65: 98-106.

27. Hansen AM, Bonke G, Larsen CJ, Yavari N, Nielsen PE, Franzyk H (2016) Antibacterial peptide nucleic acid antimicrobial peptide (PNA-AMP) conjugates: antisense targeting of fatty acid biosynthesis. Bioconjug Chem 27: 863867.

28. Valero J, Shiraishi T, de Mendoza J, Nielsen PE (2015) Cellular antisense activity of PNA-Oligo (bicycloguanidinium) conjugates forming self-assembled nanoaggregates. Chembiochem 16: 1593-1600.

\section{Corresponding author}

Anubrata, Ghosal, PhD

Department of Biology, Massachusetts Institute of Technology,

77 Massachusetts Ave, Cambridge, MA 02139

Phone: +16172533745

Fax: +1 6172538699

Email: anubrata@mit.edu

Conflict of interests: No conflict of interests is declared. 